\title{
PHÂN LOẠI LỚP PHỦ BẰNG PHƯƠNG PHÁP TIẾP CẬN HƯỚNG ĐỐI TỰ̛̣G TRÊN ẢNH SPOT LUUU VỤ๋C SUỐI MUỘ, THUẬN CHÂU, SƠN LA
}

\author{
ThS. NGUYẼN VĂN LỌ'|(1) \\ TS. VŨ KIM CHI (2) \\ (1)Trường Đại học Mỏ địa chất \\ (2) Viện Việt Nam học và Khoa học Phát triển
}

\section{Tóm tắt:}

Mục đích bài báo này nhằm kiểm chứng độ chính xác của phương pháp tiếp cận hướng đối tượng trong phân loại ảnh độ phân giải cao. Dư liệu sử dụng trong nghiên cứu này là ảnh vệ tinh SPOT5 lưu vực Suối Muội, Thuận Châu, Sơn La chụp năm 2010 với độ phân giải kênh toàn sắc đạt $2.5 \mathrm{~m}$, độ chính xác tốt với sai số tổng thể đạt $87.5 \%$, và hệ số Kappa đạt 0.85 .

\section{Giới thiệu}

Độ chính xác phân loại lớp phủ là rất quan trọng trong việc quản lý, quan trắc và quy hoạch sử dụng đất. Phương pháp phân loại truyền thống dựa vào giá trị phổ của điểm ảnh đã được sử dụng từ rất lâu, nhưng đến nay bộc lộ nhiều hạn chế về mặt thời gian và độ chính xác.... Nhằm khắc phục những hạn chế đó, phương pháp phân loại tiếp cận hướng đối tượng được đưa ra từ những năm 1970 với nhiều ưu điểm nổi bật. Nếu cụ thể hóa được, có thể chiết xuất được những đối tượng trên ảnh có liên quan mật thiết với những đối tượng ở ngoài thực địa. Khi những đối tượng này được chiết tách, mối quan hệ không gian với các đối tượng khác (ví dụ như: liền kề, chứa đựng, được chứa bởi...), bản thống kê tổng hợp về giá trị phổ, cấu trúc, và đặc điểm hình dạng đều có thể được sử dụng trong các bước phân loại [1]. Mặc dù có nhiều tiến bộ nhưng phương pháp tiếp cận hướng đối tượng vẫn còn thiên về tính toán và sự cải tiến về phân loại không phải lúc nào cũng rõ ràng. Cho đến nay, khi mà khả năng lưu trữ của phần cứng cũng như độ phân giải không gian của dữ liệu ảnh viễn thám tăng lên thì phương pháp này được ứng dụng rộng rãi với độ chính xác cao hơn hẳn so với phương pháp phân loại dựa trên điểm ảnh[3],[7],[9]. Phương pháp phân loại tiếp cận hướng đối tượng không chỉ phân biệt các loại lớp phủ và hiện trạng sử dụng đất theo giá trị phổ của các điểm ảnh mà còn xem xét đến hình dạng của đối tượng (shape), kiến trúc của đối tượng (texture) và mối quan hệ giữa các đối tượng sử dụng trong phân loại [6], [9]. Thêm vào đó, phương pháp này còn có khả năng tích hợp với các dữ liệu khác như bản đồ chuyên đề, mô hình số độ cao,... và kiến thức chuyên gia để cho ra kết quả phân loại tin cậy hơn [5], [9]. Quá trình phân loại tiếp cận hướng đối tượng bắt đầu bằng việc phân mảnh các đối tượng ảnh riêng rẽ thông qua việc gộp các pixel lân cận có mức độ đồng nhất về đặc điểm phổ, cấu trúc không gian và quan hệ không gian mà mắt người có thể nhận biết được. Sau đó, các đối tượng được phân biệt dựa trên phân loại mờ theo quy tắc phân cấp [2]. Trong bài báo này, tác giả đã xây dựng bộ quy tắc cho phân loại lớp phủ và sử dụng đất lưu vực Suối Muội, Thuận Châu, Sơn La với loại lớp phủ được xác định đó là rừng kín, rừng thưa, cây bụi, trảng cỏ, nương rẫy, đất trồng lúa nước, dân 
cư và đất mặt nước chuyên dùng. Kết quả phân loại được đánh giá độ chính xác thông qua việc kiểm chứng 32 mẫu thực địa vào năm 2013.

\section{Khu vực nghiên cứu và dữ liệu ảnh}

Lưu vực Suối Muội bao gồm địa phận thuộc 14 xã, huyện Thuận Châu và xã Chiềng Đen, thị xã Sơn La. Trung tâm của lưu vực là khu vực thị trấn Thuận Châu, cách thị xã Sơn La 30 km theo đường quốc lộ 6 về phía Tây Bắc.

Tổng diện tích đất tự nhiên lưu vực là 284 km2, trong đó đất nông nghiệp chiếm $44 \%$, trảng cỏ và cây bụi chiếm $46 \%$, đất thổ cư $4 \%$ còn lại chỉ có $6 \%$ là đất rừng (Dedobbeleer, L. 2002).

Bảng 1: Thông số ảnh SPOT5 (chụp ngày 22 tháng 8 năm 2010)

\begin{tabular}{|l|l|l|}
\hline Kênh & Tên kênh & Dải sóng \\
\hline 1 & Visiable & $0.50-0.59 \mu \mathrm{m}$ \\
\hline 2 & Visiable & $0.61-0.68 \mu \mathrm{m}$ \\
\hline 3 & Visiable & $0.78-0.89 \mu \mathrm{m}$ \\
\hline 4 & $\begin{array}{l}\text { Near- } \\
\text { Infrared }\end{array}$ & $1.58-1.75 \mu \mathrm{m}$ \\
\hline 5 & $\begin{array}{l}\text { Độ phân } \\
\text { giải }\end{array}$ & $\begin{array}{l}10 \mathrm{~m}(\text { kênh } \\
\text { Pan là } 2.5 \mathrm{~m})\end{array}$ \\
\hline
\end{tabular}

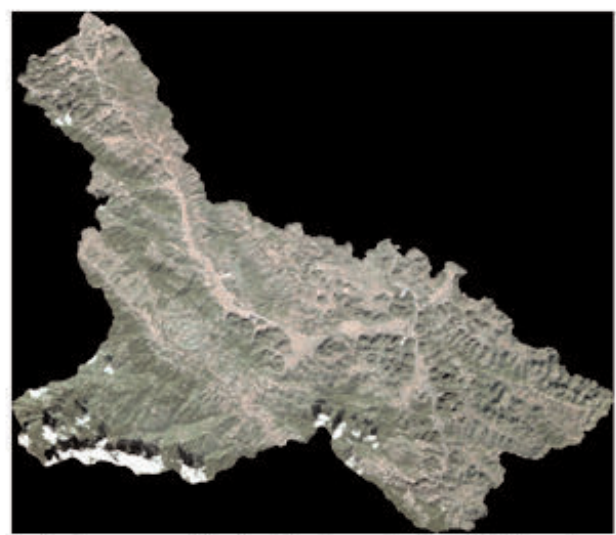

Hinh 1. Iuu vuc Suối Muội, Thuân Châu, Son La

\section{Phương pháp nghiên cứu}

Phương pháp phân loại tiếp cận gồm 2 giai đoạn chính là phân mảnh ảnh và phân loại ảnh theo quy tắc mờ.

\subsection{Phân mảnh ảnh}

Ảnh vệ tinh lưu vực Suối Muội được phân mảnh thành các đối tượng ảnh dựa vào thuật toán segmentation bao gồm 2 cấp: cấp 1, phân mảnh đa độ phân giải (multiresolution segmentation) và cấp 2, phân mảnh dựa vào sự khác biệt phổ (spectral difference segmentation). Cấp 1, tác giả đã lựa chọn các thông số như sau: thông số tỷ lệ (scale parameter: 30), hình dạng (shape: 0.5), màu sắc (color: 0.5 ), độ chặt (compactness: 0.5), độ trơn (smoothness: 0.5), trọng số (image layer weights: 1.1.1). Với cấp 2, các chỉ tiêu đã được chọn là Sự khác biệt phổ lớn nhất (maximum spectral difference: 5), trọng số (image layer weights: 1.1.1). (Xem hình 2)

\subsection{Phân loại ảnh theo quy tắc mờ}

3.2.1. Phân bậc đối tượng ảnh vệ tinh lưu vực Suối Muội. (Xem hình 3)

3.2.2. Xây dựng bộ quy tắc (rule set) phân loại ảnh. (Xem hình 4)

Các yếu tố trên ảnh SPOT5 được chiết tách chủ yếu từ chỉ số thực vật NDVI (normal different vegetatation indicate), các kênh phổ (layer 3 - red, layer 2 - NIR), các chỉ số khác như density, area.

Trong đó chỉ số NDVI được tính như sau: $N D V I=\frac{N I R-\operatorname{Re} d}{N I R+\operatorname{Re} d}, \quad \mathrm{NDVI}$ nằm trong khoảng $[-1 ; 1]$.

\subsubsection{Phân loại (theo quy tắc mờ)}

Bước 1: Mờ hóa, phân chia các đối tượng đất, nước, thực vật theo các khoảng phổ khác nhau bằng thuật toán assign class. Dễ dàng nhận thấy là có nhiều pixel có giá trị phổ giống nhau nhưng thực tế lại 


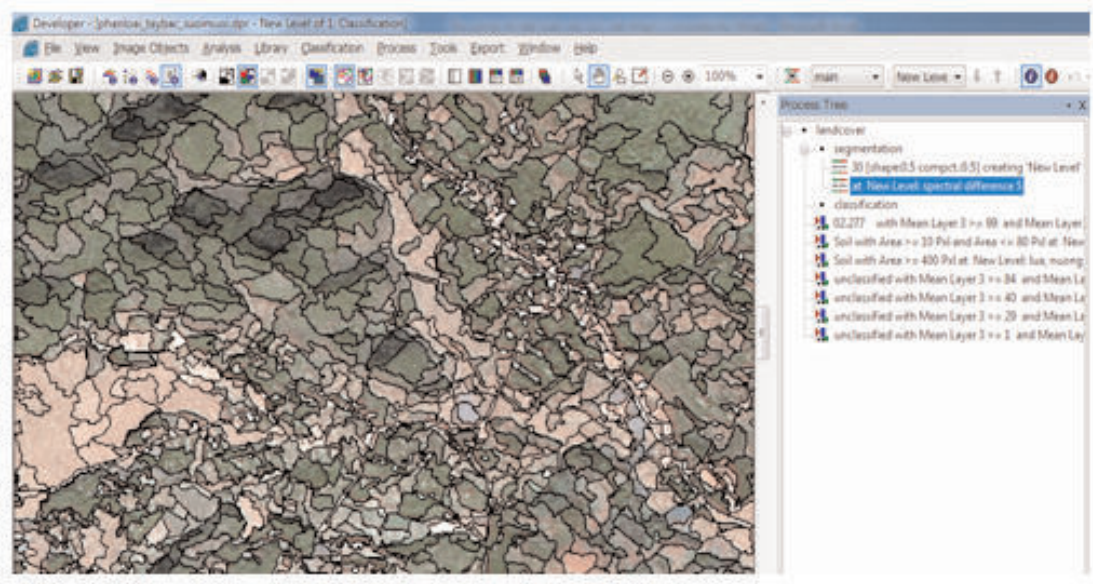

Hình 2: Kết quả phân mảnh ảnh

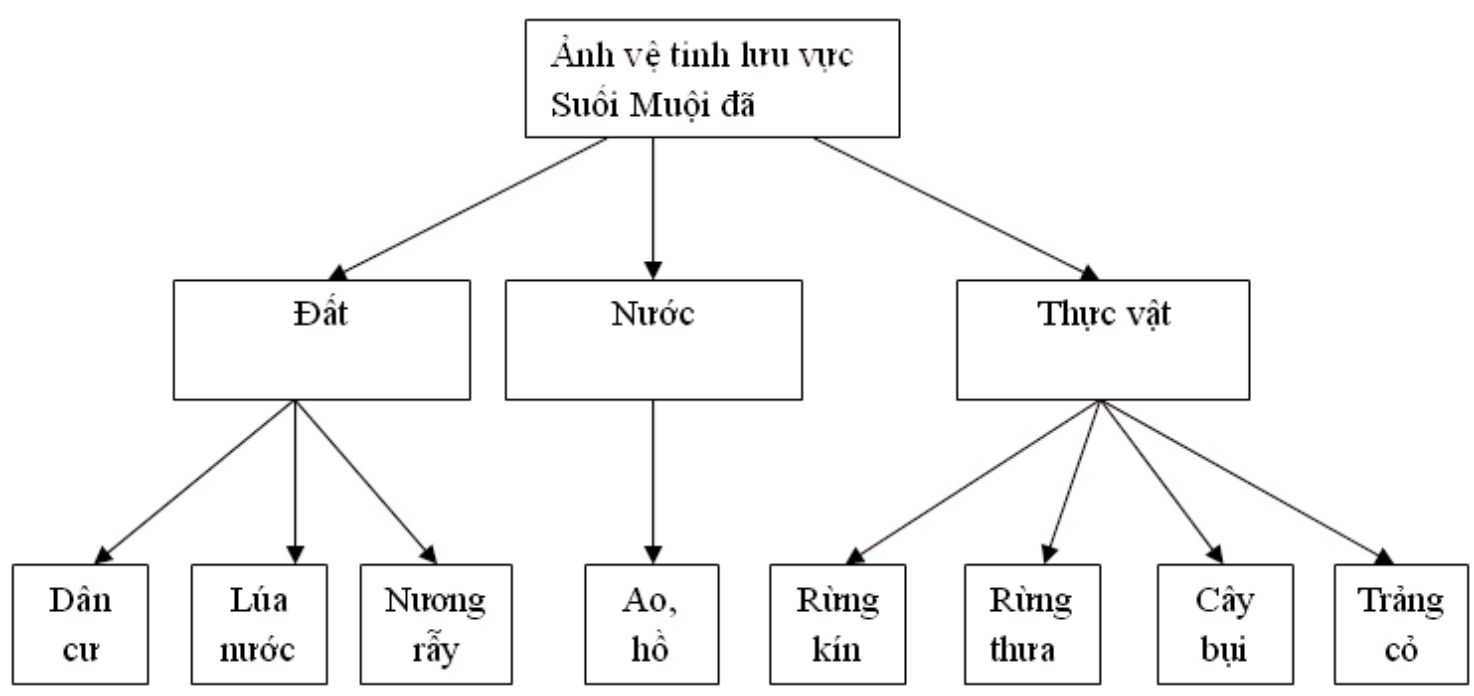

Hình 3: Sơ đồ phân bậc đối tượng ảnh vệ tinh Suối Muội năm 2010

là những đối tượng khác nhau. Vì vậy, tác giả đã khảo sát giá trị phổ của các đối tượng trên ảnh và đưa ra được vùng giá trị phổ giao nhau giữa các đối tượng (vùng mờ), quá trình này gọi là mờ hóa. Như vậy, những pixel thuộc những đối tượng khác nhau nhưng có giá trị phổ giống nhau sẽ được làm mờ hóa.

Bước 2: Suy luận mờ, quá trình này tác giả đã suy luận logic về mối liên hệ địa lý của các yếu tố, các chỉ số chiết tách thực vật NDVI để đưa thông tin vào vùng mờ phục vụ cho việc khử mờ. Cụ thể với ảnh SPOT5 Suối Muội đã được đưa thêm các chỉ số về NDVI, diện tích (Area), mật độ (density), độ chặt (compatchness).

Bước 3: Khử mờ, trong bước khử mờ tác giả đã khảo sát và lựa chọn hàm liên thuộc (membership function) là hàm có hình tháp chuông. (Xem hình 5) 


\section{Anh SPOTS Suôi Muội}

1. multivesolution segmentation, shape (05), corrquactres (05)

2. spectral difference segmentation, spectral difference (5)

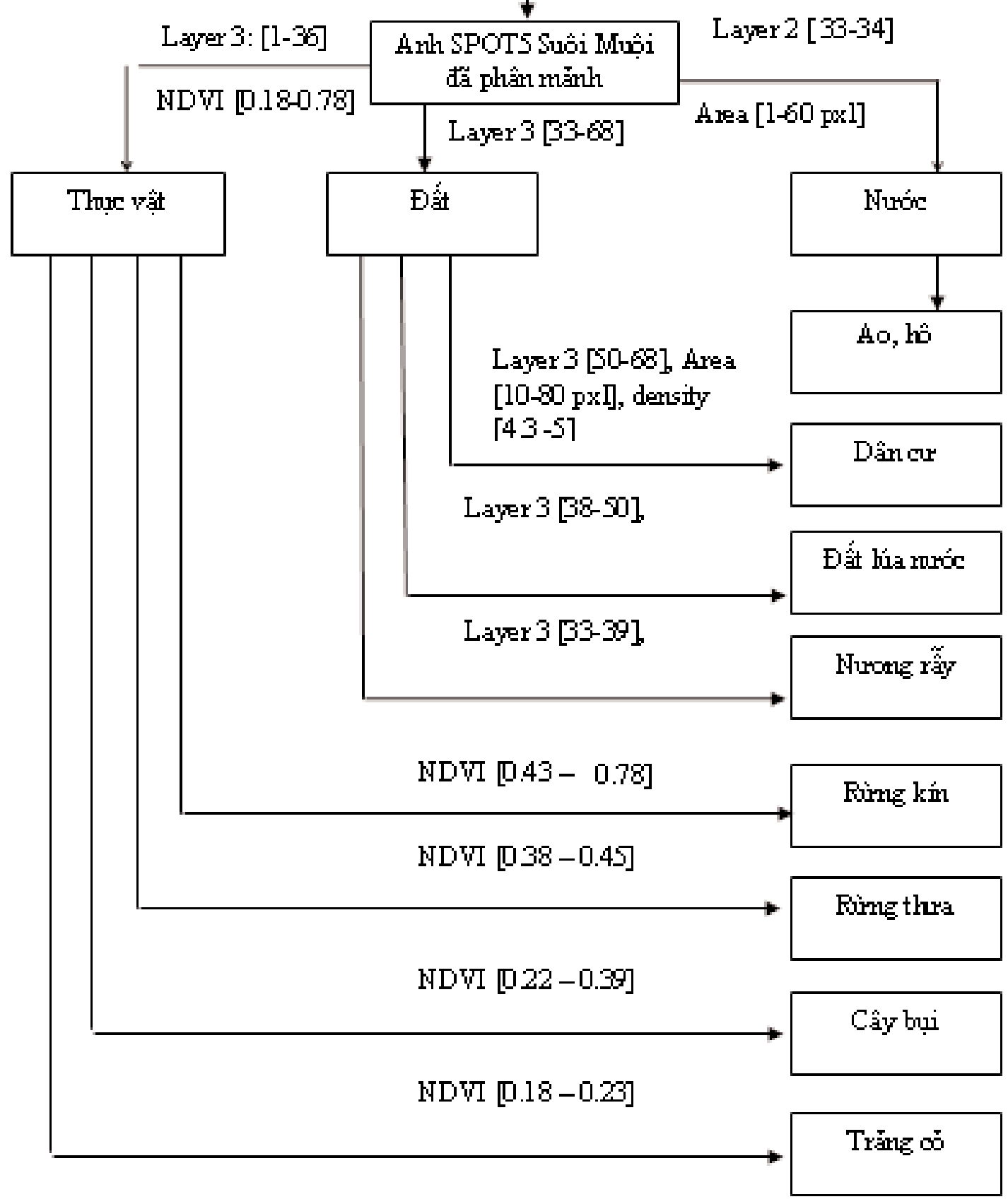

Hình 4: Chiết xuất các đối tượng bằng phương pháp tiếp cận hướng đối tượng 


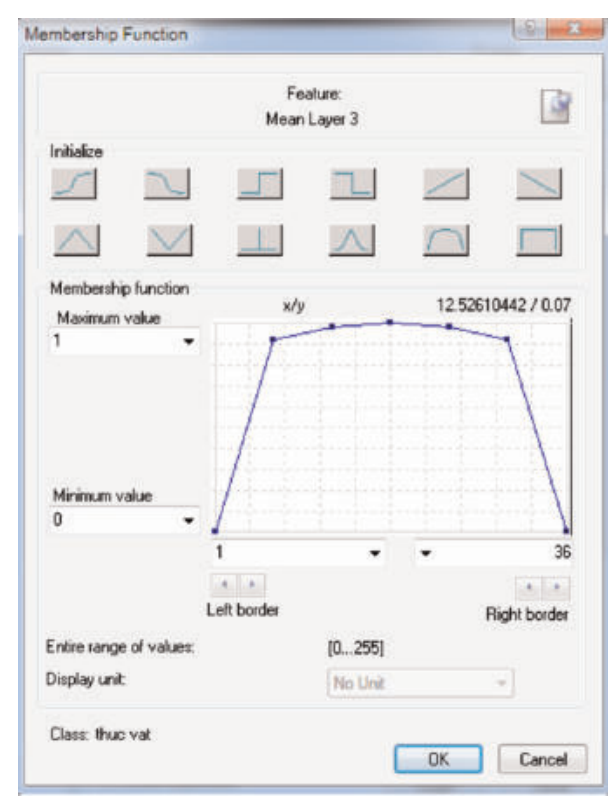

Hình 5: Lựa chọn hàm liên thuộc

\section{Kết quả và đánh giá độ chính xác}

Cụ thể với việc phân loại ảnh lưu vực Suối Muội, tác giả đã trực tiếp ra thực địa thu thập được 32 mẫu đối chứng với kết quả phân loại, và thành lập ma trận sai số. Độ chính xác được đánh giá bằng sai số tổng thể và hệ số Kappa thông qua ma trận sai số. Cụ thể, sai số tổng thể đạt $87.5 \%$, hệ số Kappa đạt 0.85 là rất cao. (Xem bảng 2, hìn 6)

Kết quả phân loại đạt yêu cầu để thành lập bản đồ hiện trạng sử dụng đất lưu vực Suối Muội, Thuận Châu, Sơn La.

\section{Kết luận}

Phân loại lớp phủ dựa trên phương pháp tiếp cận hướng đối tượng với logic mờ, có ưu điểm là không chỉ dựa vào giá trị phổ tổng hợp các kênh ảnh mà có thể sử dụng riêng rẽ đặc tính của từng kênh ảnh để phân biệt các lớp sử dụng đất và lớp phủ dựa vào quy tắc phân cấp các đối tượng. Bên cạnh đó, việc phân loại còn sử dụng kết hợp các đặc điểm của đối tượng như hình dạng, kích thước, diện tích ....nhằm đem lại kết quả tốt hơn. Kết quả phân loại cho thấy phương pháp phân loại này tốt cho hầu hết các lớp đối tượng lớp phủ.
Độ chính xác của phân loại phụ thuộc vào độ chính xác của quá trình phân mảnh ảnh và quá trình xác định rule set cho việc phân loại. Kết quả phân mảnh ảnh càng tốt thì độ chính xác của quá trình phân loại càng cao và ngược lại.

Nghiên cứu này được tài trợ bởi Quỹ phát triển khoa học và công nghệ quốc gia (NAFOSTED) trong đề tài mã số 105.062012.20. 0

\section{Tài liệu tham khảo}

[1]. Benz, U.C., P. Hofmann, G. Willhauck, Lingenfelder and $M$. Heynen (2003), "Multiresolution, object-oriented fuzzy analysis of remote Sensing data for GIS-ready information", Journal of Photogrametry and Remote Sensing,58, pp.239-258.

[2]. Definiens (2007a, b) Definiens Imaging Developer 7. eCognition Software

[3]. Michael Bock, Panteleimon Xofis, Jonathan Mitchley, Godela Rossner, and Michael Wissen (2005), "Object-oriented methods for habitat mapping at multiple scales - Case studies from Northern Germany and Wye Downs, UK", Journal for Nature Conservation, 13, pp. 75-89.

[4]. Hanqiu Xu (2008), "A new index for delineating built-up land features in satellite imagery", International Journal of Remote Sensing, 29, pp. 4269-4276.

[5]. Heck R. J Dehvari A (2009), "Comparison of object based and pixel based infrared airborne image classification methods using DEM thematic layer", Journal of Geo"graphy and Regional Planning, 2, pp. 86 - 96.

[6]. Prasun K. G Gaurav K.P (2010), "Comparison of Advanced Pixel based (ANN and SVM) and Object oriented classification approaches using Landsat 7 ETM data", International Journal of Engineering and Technology, 2, pp. 245 - 251.

[7]. D. Geneletti, and B. G. H. Gorte (2003), "A method for object-oriented land cover classification combining Landsat TM 
Bảng 2: Ma trận sai số phân loại ảnh Suối Muội

\begin{tabular}{|l|c|c|c|c|c|c|c|c|c|}
\hline & $\begin{array}{c}\text { Nương } \\
\text { rẫy }\end{array}$ & $\begin{array}{c}\text { Đất lúa } \\
\text { nước }\end{array}$ & Trảng cỏ & Cây bụi & $\begin{array}{c}\text { Rừng } \\
\text { thưa }\end{array}$ & Rừng kín & Dân cư & Ao, hồ & Tổng \\
\hline Nương rẫy & 4 & 0 & 0 & 0 & 0 & 0 & 0 & 0 & 4 \\
\hline Đất lúa nước & 1 & 3 & 0 & 0 & 0 & 0 & 0 & 0 & 4 \\
\hline Trảng cỏ & 1 & 1 & 2 & 0 & 0 & 0 & 0 & 0 & 4 \\
\hline Cây bụi & 0 & 0 & 0 & 4 & 0 & 0 & 0 & 0 & 4 \\
\hline Rừng thưa & 0 & 0 & 0 & 0 & 4 & 1 & 0 & 0 & 5 \\
\hline Rừng kín & 0 & 0 & 0 & 0 & 0 & 3 & 0 & 0 & 3 \\
\hline Dân cư & 0 & 0 & 0 & 0 & 0 & 0 & 5 & 0 & 5 \\
\hline Ao, hồ & 0 & 0 & 0 & 0 & 0 & 0 & 0 & 3 & 3 \\
\hline Tổng & 6 & 4 & 2 & 4 & 4 & 4 & 5 & 3 & \\
\hline
\end{tabular}

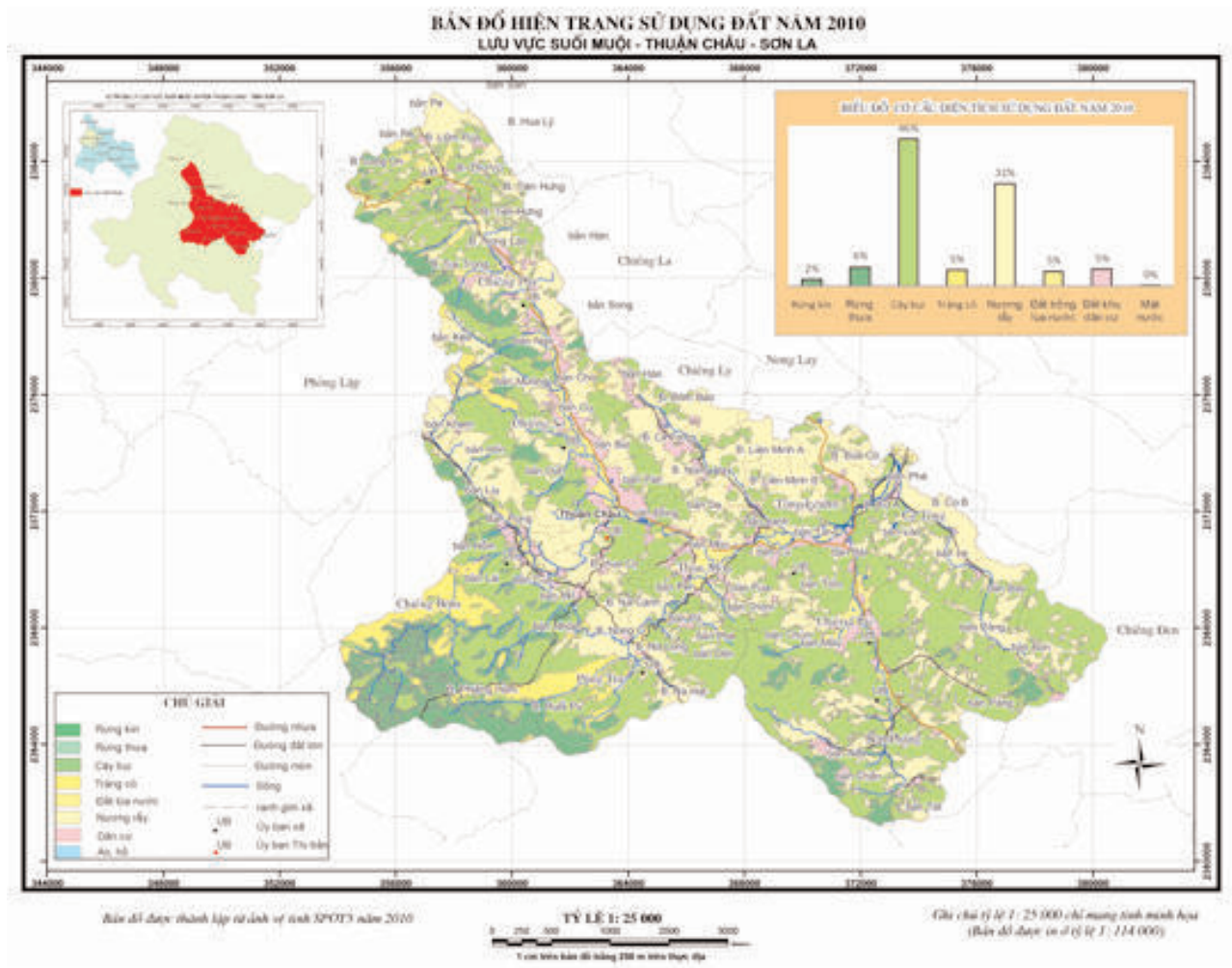

Hình 6: Bản đồ hiện trạng sử dụng đất lưu vực Suối Muội năm 2010 
data and aerial photographs", International Journal of Remote sensing, 24, pp. 12731286.

[8]. Ray D. Jackson, Fredo R. Huete (1991), "Interpreting vegetation indices", Preventive Veterinary Medicine, 11, pp. $185-200$.
[9]. Urszula Janas, Aleksandra Mazur, and Jacek Andrzej Urbański (2009), "Object-oriented classification of QuickBird data for mapping seagrass spatial structure", Oceanological and Hydrobiological Studies, 38, pp. 27-43. 0

\section{Summary}

Cover classification by object-oriented approach methods on SPOT images in Suoi Muoi basin, Thuan Chau district, Son La province

MSc. Nguyen Van Loi, Hanoi University of Mining and Geology,

Dr. Vu Kim Chi, Institute of Vietnamese Studies and Development Sciences

The purpose of this paper to verify the accuracy of the object-oriented approach methods in classification of high-resolution images. The data used in this study is the SPOT5 satellite images Suoi Muoi basins, Thuan Chau, Son La, taken in 2010 with a resolution panchromatic channel reaches $2.5 \mathrm{~m}$, with accuracy overall error reached $87.5 \%$, and Kappa coefficient reached 0.85.m

Ngày nhận bài: 18/3/2014.

\section{ĐÁNH GIÁ Độ CHÍNH XÁC.....}

\section{(Tiếp theo trang 13)}

[19]. Pelton J.R. and Smith R.B. (1979). Recent crustal uplift in Yellowstone National Park, Science, V. 206, p. 1179-1182.

[20]. Reilinger R.E., Oliver J.E., Brown L.D., Sanford A.R. and Balazs E.I. (1980). New measurements of crustall doming over the Socorro magma bogy. Geology, V.8, p. 291-295, New Mexico.

[21]. Reilinger R.E. (1980). Elevation changes near the San Gabriel Fault, Southern California. Geophysical Research Letters, V. 7, No 11, pp. 1017-1019.

[22]. Thomas Gruber (2008). Evaluation of the EGM2008 Gravity field by Mean of GPSLevelling and Sea surface topography solution. Institute of Astronomical and Physical Geodesy, Technical University of Munich, Germany. E-mail: Thomas.Gruber@bv.tumuenchen.de. $\mathrm{O}$

\section{Summary}

\section{Assessment of accuracy of the EGM2008 Quasigeoid on the territiry of Vietnam}

Assoc. Prof. Dr. Sc. Ha Minh Hoa, Vietnam Institute of Geodesy and Cartography

This scientific article assessed accuracy of a local gravimatric quasigeoid model created from the EGM2008 and transfered to the quasigeoid Hon Dau. Comparing to local GPS/leveling quasigeoid created from 185 GPS/leveling height anomalies on 185 first order vertical benchmarks, standard deviation of the local gravimatric quasigeoid is $\pm 8,5$ $\mathrm{cm}$, differences between GPS/leveling and gravimetric height anomalies are in the range between - 0,224 $\mathrm{m}$ and 0,236 m. $\mathrm{O}$

Ngày nhận bài: 20/5/2014. 